\title{
Radioulnar Ischaemic Necrosis in a Dog with Concurrent Osteomyelitis and Bone Sequestration
}

\author{
Theresa L. Aller ${ }^{1}$ Kathryn L. Phillips ${ }^{2}$ Amy S. Kapatkin ${ }^{2}$ Katherine D. Watson ${ }^{3}$ \\ ${ }^{1}$ Department of Diagnostic Imaging, University of California Davis, \\ Davis, California, United States \\ ${ }^{2}$ Department of Surgical and Radiological Sciences, University of \\ California Davis, Davis, California, United States \\ ${ }^{3}$ Department of Pathology, California Animal Health and Food Safety \\ Laboratory System, Davis, California, United States \\ Address for correspondence Theresa L. Aller, DVM, Pet Emergency \\ and Specialty Center, San Diego, CA, 91942, United States \\ (e-mail: tlaller@ucdavis.edu). \\ VCOT Open 2020;3:e19-e22.
}

\begin{abstract} Keywords

- radioulnar ischaemic necrosis

- osteonecrosis

- haematogenous osteomyelitis

- sequestrum

An 11-month-old intact female Golden Retriever presented for a 3- to 4-week history of right thoracic limb lameness. Computed tomography of the limb showed a sequestrum with a periosteal and endosteal reaction at the level of the nutrient foramen of the radius. Septic osteomyelitis was suspected based on cytology. Repeat examination and imaging revealed improvement in the lesion and resolution of the lameness. This lesion is similar to reports of radioulnar ischaemic necrosis, but is the first report with concurrent osteomyelitis and sequestration.
\end{abstract}

\section{Introduction}

Radioulnar ischaemic necrosis (RUIN) is a term that has been used to describe osteonecrosis secondary to ischaemia of the radius and ulna in the dog. ${ }^{1}$ There is a small amount of data in the literature describing the radiographic appearance of a RUIN lesion. ${ }^{1}$ Osteonecrosis in dogs has also been reported to occur with Legg-Calves-Perthes, idiopathic osteonecrosis of the carpal bone and maxillofacial osteonecrosis. ${ }^{2-4}$ This is the first case report of concurrent haematogenous osteomyelitis and ischaemic necrosis occurring at the site of a lesion that is radiographically consistent with RUIN.

\section{History and Clinical Findings}

An 11-month-old intact female Golden Retriever presented to the Veterinary Medical Teaching Hospital of the University of California - Davis, School of Veterinary Medicine for evaluation of a nontraumatic right thoracic limb lameness. The patient had a 3-4 week history of waxing and waning lameness that had been managed with a combination of carprofen $(3.9 \mathrm{mg} / \mathrm{kg}$ per os [PO] SID), tramadol (unknown dose) and cage rest. The

received

June 18, 2019

accepted after revision

January 7,2020
DOI https://doi.org/

10.1055/s-0040-1702152. ISSN 2625-2325. referring veterinarian suspected panosteitis due to the lack of overt radiographic findings in the right thoracic limb. On examination, the dog was bright, with vital parameters within normal limits, a body condition score of $4 / 9$ and a weight of $25.5 \mathrm{~kg}$. The patient had a marked right thoracic limb lameness with decreased weight bearing function, moderate ipsilateral muscle atrophy and subtle right carpal valgus. The patient was painful during shoulder extension, and on long-bone palpation of the right radius, right ulna and bilaterally along the femurs. The referring veterinarians' radiographs were reviewed by a radiologist, deemed of good quality, and a definitive cause for the lameness was not detected.

\section{Imaging, Diagnosis and Outcome}

A computed tomography (CT) scan revealed focal lysis of the proximal right radius with a large thin sequestrum and marked endosteal and periosteal new bone formation centred at the nutrient foramen (-Fig. 1). The contralateral limb CT was within normal limits. The imaging characteristics of the right thoracic limb were suggestive of osteomyelitis and additional diagnostics were performed, including an ophthalmic fundic
License terms

Stuttgart · New York

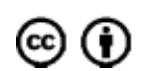



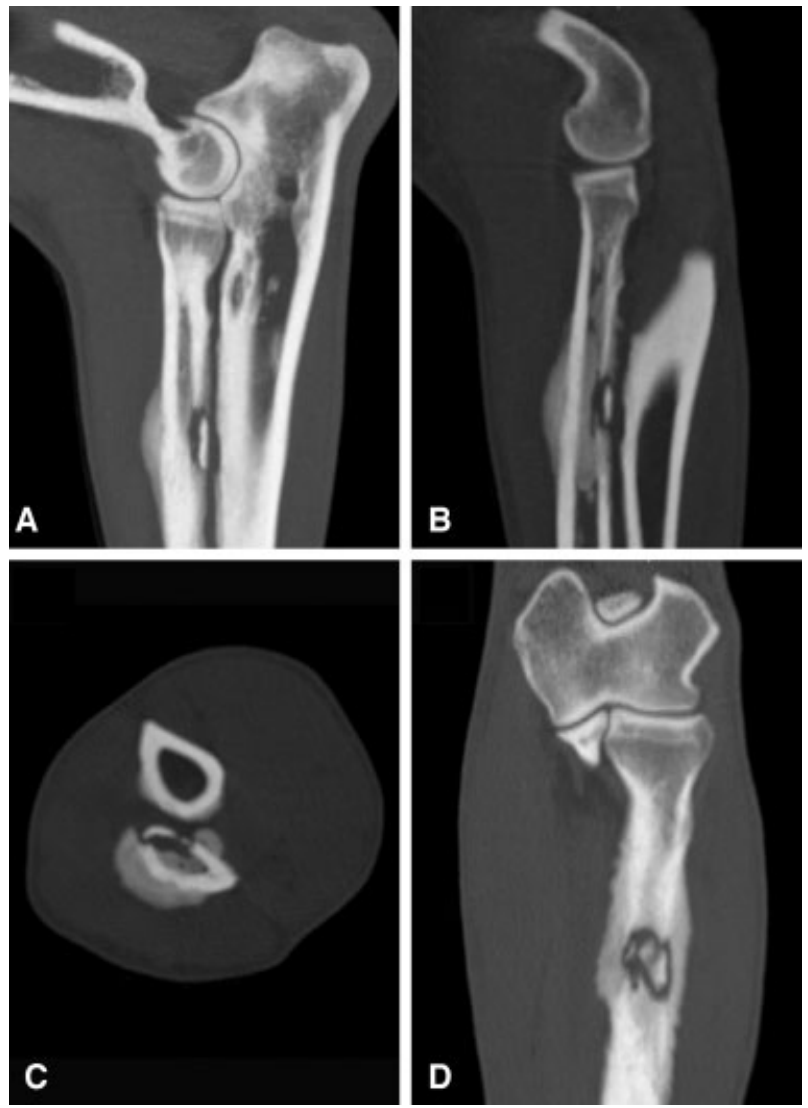

Fig. 1 Computed tomographic images of a sharp (bone) kernel of the right thoracic limb. (A and B) Sagittal multiplanar reconstructions demonstrating a thin bone sequestrum along the caudal radial cortex with smooth periosteal reaction along dorsal cortical margin and extensive new endosteal new bone formation. (C) A transverse plane at the level of the sequestrum. (D) A dorsal plane of the sequestrum in the radius.

examination, haematology, chemistry panel, urinalysis, urine culture and thoracic radiographs, all of which were unremarkable. A fine-needle aspirate from the region adjacent to the right radial sequestrum in the region of the involucrum had marked suppurative, septic inflammation featuring rodshaped bacteria. The sample was highly cellular and comprised mostly of neutrophils that were often karyolitic with phagocytized rod-shaped bacteria and fewer variably vacuolated macrophages. Aerobic, anaerobic and fungal cultures of the bone aspirate were negative, which may be attributed to a low volume sample.

The patient was sent home on antibiotic medications (Clavamox; $14.7 \mathrm{mg} / \mathrm{kg}$ PO q12 $\times 14$ days) with a recommendation for strict cage rest and continuation of carprofen (3.9 mg/kg PO q24 as needed for pain). Re-evaluation with potential surgical removal of the sequestrum was discussed.

On Day 24, the dog was improving clinically at home. Reevaluation revealed she was mildly lame on the right thoracic limb with mild, nonpainful swelling over her right radius. Radiographs were acquired at this recheck evaluation and the osseous sequestrum was not detected. A recheck sedated CT scan was performed for further investigation and showed a healing lytic lesion of the radial diaphysis, and resolution of
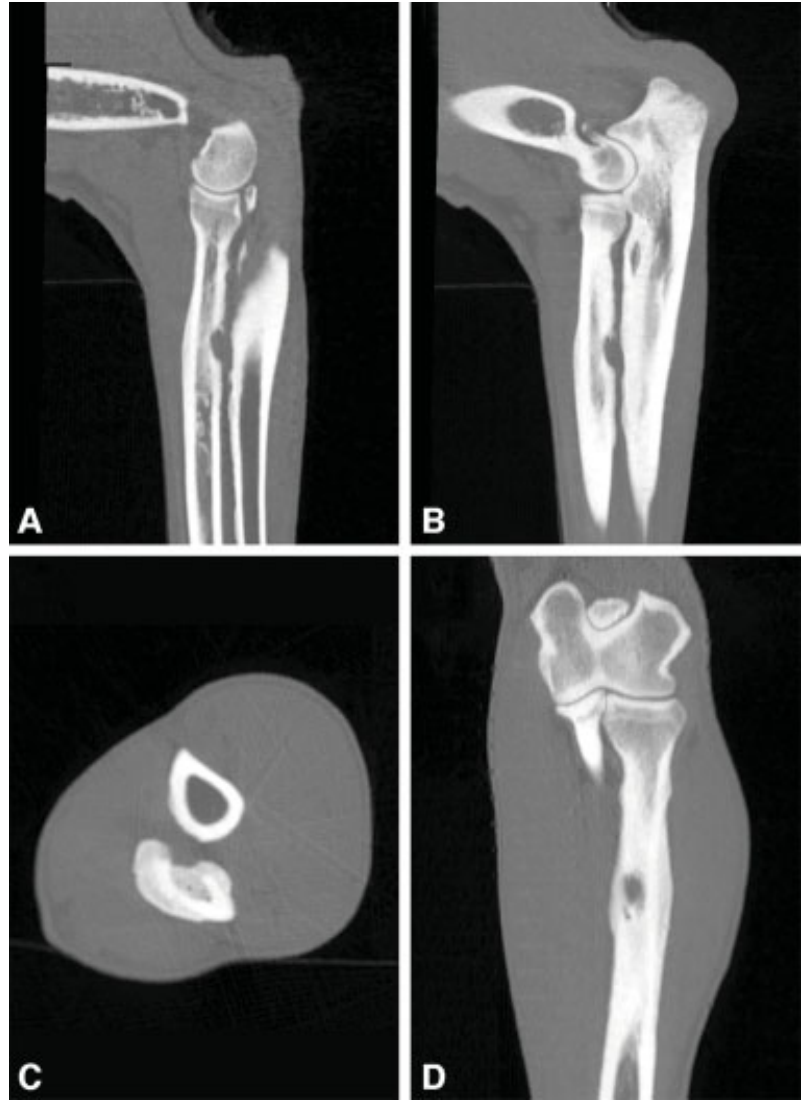

Fig. 2 Computed tomographic images 24 days after presentation of the right thoracic limb. (A and B) Sagittal multiplanar reconstructions at the level of the previous sequestrum. The sequestrum is no longer visualized and a concave region of lysis persists in the caudal radial cortex at the level of the nutrient foramen. (C) A transverse plane showing the concave lysis of the caudal radial cortex with severe endosteal new bone and smooth periosteal reaction. (D) A dorsal plane of the caudal radial cortex.

the sequestrum (-Fig. 2). Since the dog was clinically improving and imaging showed a resolving lesion, surgery was no longer indicated. Continued exercise restriction, antibiotic medication and trazodone $(5.8 \mathrm{mg} / \mathrm{kg} \mathrm{PO}$ q12 as needed) were recommended.

On day 41 , the dog had a recheck examination. She was no longer lame on physical examination, and had no pain elicited on long bone palpation over the lesion previously identified on her right radius and ulna. A CT was performed. The lesion had continued to resolve with smooth margins, milder periosteal reaction and markedly reduced medullary sclerosis ( - Fig. 3). The patient was discharged and advised to resume physical activity since she was clinically normal and the CT demonstrated improvement in the osteolytic lesion.

\section{Discussion}

Bone sequestration is a radiographic and pathological finding more commonly described in large animals than in small animals. ${ }^{5,6} \mathrm{~A}$ bone sequestration is pathologically defined as a nonviable fragment of bone that has lost its blood supply through a necrotic process. Radiographically, it manifests as a sharply marginated sclerotic bone fragment surrounded by a 

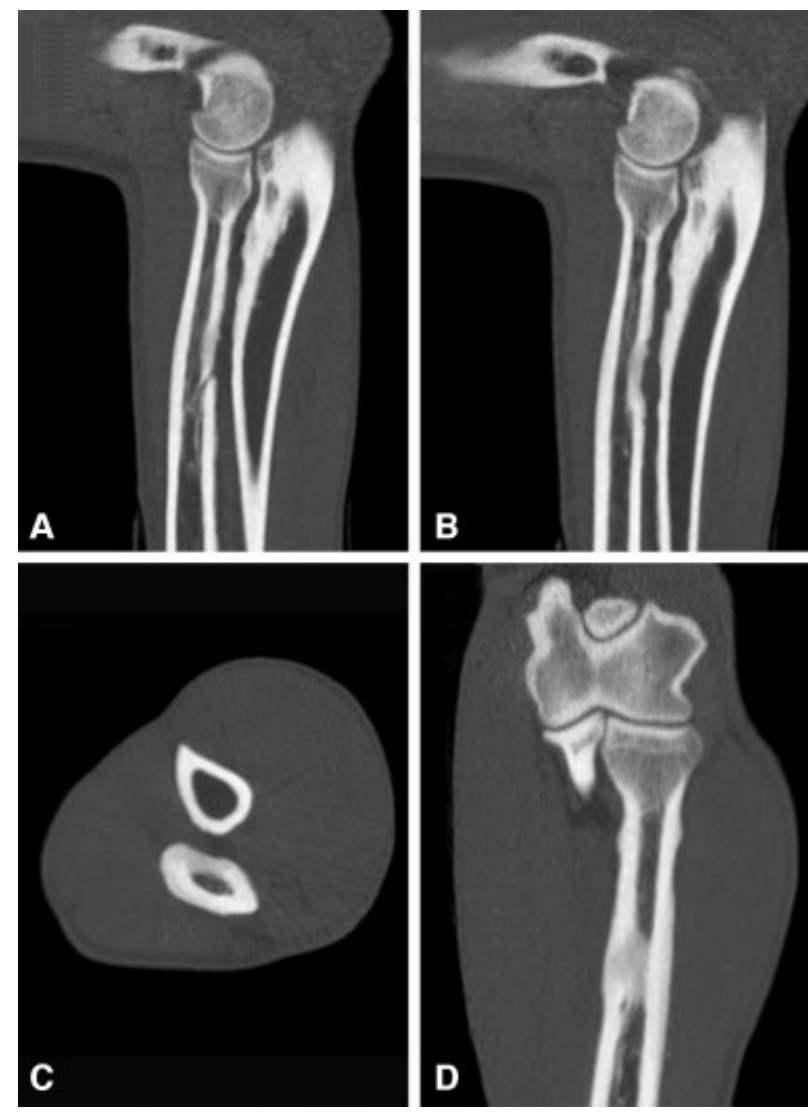

Fig. 3 Computed tomographic images 41 days after initial presentation of the right thoracic limb shows great improvement in the lesion. (A and B) Sagittal multiplanar reconstructions, a concave divot persists in the caudal radial cortex at the level of the nutrient foramen, the endosteal sclerosis has greatly improved. (C) A transverse plane. (D) A dorsal multiplanar reconstruction.

radiolucent zone. Bone sequestra can be sterile or infectious and can sometimes involve a draining tract. ${ }^{7}$ In dogs, formation of sequestra is most often associated with osteomyelitis following traumatic fractures and/or an orthopaedic surgical complication. ${ }^{8-12}$

In this instance, osteomyelitis was diagnosed based on the imaging features of an aggressive bone lesion and the presence of septic suppurative inflammation in the involucrum. Fineneedle aspirates are minimally invasive and have been successfully used to diagnose bony lesions in both veterinary and human medicine..$^{2,13-17}$ Although histopathology from a surgical biopsy could have strengthened our diagnosis, it was not performed due to the positive clinical response in the recheck interim and the difficulty of reaching the lesion.

Osteomyelitis is an infection of the bone and marrow occurring from three main pathogeneses: haematogenous, contiguous and direct inoculation. ${ }^{18}$ In dogs, osteomyelitis most commonly occurs from direct inoculation of bacteria and fungi from an open wound or after osteosynthesis; however, haematogenous spread with seeding at the vascular metaphyses of long bones and near the nutrient foramen has also been described. ${ }^{19,20}$ The lack of a history of trauma and the location of the lesion centred at the nutrient foramen of the radius in this case make haematogenous osteomyelitis most likely.
Osteonecrosis, ischaemic necrosis and avascular necrosis are all terms used to describe the process of cell death caused by loss of blood supply to the bone. ${ }^{21,22}$ Radioulnar ischaemic necrosis is defined as osteonecrosis of the radius and/or ulna in the dog due to ischaemic injury of unknown aetiology. ${ }^{1}$ Radiographically, RUIN lesions appear as osteolytic lesions on the opposing radial and ulnar cortices. The caudal interosseous artery feeds the intramedullary blood supply of both the radius and ulna. The radial nutrient foramen, which is typically evident on lateral radiographs, lies along the caudal cortical margin approximately $1 / 3$ the way down the diaphysis. The ulnar nutrient foramen is through the opposing dorsal cortical margin just proximal to the radial nutrient foramen at about the level where the radius and ulna become superimposed on a lateral radiograph.

This is also the region of the interosseous ligament, which spans the space between the radius and ulna. The interosseous ligament has a wide insertion along the proximal $1 / 3$ of the radius from the nutrient foramen proximally to a wider attachment along the proximal $1 / 2$ of the ulna. Frequently, there is enthesis new bone at the insertion of the interosseous ligament creating an irregular margin to the opposing cortices. This insertional desmopathy confounds interpretation of this region because it is not uncommonly seen in dogs without lameness. ${ }^{23}$ Concavities or lucencies have been described at the insertion of the interosseous ligament varying in size from 2 to $10 \mathrm{~mm} .^{24}$ The osseous changes seen with interosseous desmopathy are indicative of chronic or previous change. While a definitive cause for the concavities at the insertion of the interosseous ligament is unknown, one proposed hypothesis is previous desmopathy of the interosseous ligament in a juvenile patient that altered normal radial widening of the bone in this region while the adjacent bone grew normally. This hypothesis presumes an alteration in the blood supply at the site of the interosseous insertion. ${ }^{24}$

The terminology of RUIN has been applied to describe these concave lucencies along apposing margins of the radius and ulna regardless of the pathophysiology whether these lesions may be a manifestation of chronic interosseous desmopathy or any cause for ischaemia. In this case, the patient presented with a sequestrum, osteolysis with marked endosteal and periosteal reaction centred at the nutrient foramen of the proximal right radius. A haematogenous osteomyelitis was the primary differential given the sequestrum, location and appearance. Cytology findings support a bacterial aetiology with septic inflammation and rod-shaped bacteria. However, the location also fits with a RUIN lesion, and on subsequent imaging studies the lesion had characteristics similar to those described for a RUIN lesion with smoothly margined lysis of the caudal radial cortex and adjacent smooth periosteal new bone formation.

Interestingly both RUIN lesions and sequestra form due to ischaemia of the bone, implying that these lesions are not exclusive entities. This case appears to be an example of RUIN in conjunction with osteomyelitis and subsequent bone sequestration. A transient bacteraemia prior to presentation to XXX causing haematogenous osteomyelitis in the radius and ulna is suspected given the nutrient foramen involvement. The inflammatory response of the bone to a haematogenous 
infection could cause damage to the supplying arteries disrupting the blood supply to the surrounding bone. Alternatively, a region of ischaemia has a degree of immune privilege that can favour bacterial growth. Pathogenesis of osteonecrosis and formation of a sequestrum have a lot of similarities supporting the idea that they may occur simultaneously. Both are caused by disruption of blood supply leading to cell necrosis. Radiographically, both involve a lucent region, the only difference being that sequestra have a central piece of sclerotic bone within the lucent rim. Thus, it makes sense to propose in this case that their pathogeneses were linked.

The purpose of this case study was to present another case example of RUIN as there is a paucity of published information in the literature. We postulated an aetiology other than trauma and chronic desmopathy as a cause for RUIN lesions. In the present case, there is evidence of an infectious aetiology, given the presence of a sequestrum, the results of the fine-needle aspirate and the clinical response to antibiotic medications. Whether the infection was a primary or a secondary remains unclear, but the lack of corresponding soft tissue swelling and the close proximity of a nutrient foramen suggest haematological seeding.

A larger case series or retrospective study of cases with correlated pathological and clinical findings is needed to better understand the aetiology of RUIN and the clinical impact it may have on dogs. This is complicated by the fact that many of the patients that are diagnosed with RUIN lesions radiographically have no lameness; there is great variability to the appearance of degenerative change at the insertion of the interosseous ligament that may or may not have associated osteonecrosis and as this case demonstrates these lesions can remodel and change over time.

\section{Authors' Contributions}

T.A. and K.P. contributed to the conception of study, study design, acquisition of data, data analysis and interpretation and drafting or revising of manuscript. A.K. contributed to the acquisition of data, data analysis and interpretation and drafting or revising of manuscript. K.W. contributed to acquisition of data and data analysis and interpretation. All the authors approved the submitted manuscript and agree to be accountable for all relevant content of the work.

\section{Funding}

None.

\section{Conflict of Interest}

None declared.

\section{Acknowledgments}

The authors are grateful to the students and staff of the Veterinary Medical Teaching Hospital of the University of California-Davis School of Veterinary Medicine.

\section{References}

1 Schmid L, Klang A, Katic N, Ansón A, Gumpenberger M, Kneissl S. Imaging diagnosis-radiography and computed tomography of radioulnar ischemic necrosis in a Jack Russell Terrier. Vet Radiol Ultrasound 2018;59(01):E7-E11

2 Harris KP, Langley-Hobbs SJ. Idiopathic ischemic necrosis of an accessory carpal bone in a dog. J Am Vet Med Assoc 2013;243(12): 1746-1750

3 Willems WF, Alberton GM, Bishop AT, Kremer T. Vascularized bone grafting in a canine carpal avascular necrosis model. Clin Orthop Relat Res 2011;469(10):2831-2837

4 Peralta S, Arzi B, Nemec A, Lommer MJ, Verstraete FJ. Non-radiationrelated osteonecrosis of the jaws in dogs: 14 Cases (1996-2014). Front Vet Sci 2015;2(05):7. Doi: 10.3389/fvets.2015.00007

5 Moens Y, Verschooten F, Moor D, Wouters L. Bone sequestration as a consequence of limb wounds in the horse. Vet Radiol 1980;21 (01):40-44

6 Firth EC. Bone sequestration in horses and cattle. Aust Vet J 1987; 64(03):65-69

7 Henry GA. Fracture healing and complications. In: Thrall DE, ed. Textbook of Veterinary Diagnostic Radiology. 6th ed. St Louis: Elsevier Saunders; 2013:283-306

8 Stead AC. Osteomyelitis in the dog and cat. J Small Anim Pract 1984;25(01):1-13

9 Seguin B, Harari J, Wood RD, Tillson DM. Bone fracture and sequestration as complications of external skeletal fixation. J Small Anim Pract 1997;38(02):81-84

10 Johnson KA, Lomas GR, Wood AK. Osteomyelitis in dogs and cats caused by anaerobic bacteria. Aust Vet J 1984;61(02):57-61

11 Vaughan LC. Complications associated with the internal fixation of fractures in dogs. J Small Anim Pract 1975;16(07):415-426

12 Hayes G, Harris K, Langley-Hobbs S. Post-traumatic septic bone sequestration in three dogs. VCOT Open 2019;02(02):e9-e15

13 de Farias MR, Werner J, Ribeiro MG, et al. Uncommon mandibular osteomyelitis in a cat caused by Nocardia Africana. BMC Vet Res 2012;8(01):239-243

14 Sabattini S, Renzi A, Buracco P, et al. Comparative assessment of the accuracy of cytological and histologic biopsies in the diagnosis of canine bone lesions. J Vet Intern Med 2017;31(03):864-871

15 Howard CB, Einhorn M, Dagan R, Yagupski P, Porat S. Fine-needle bone biopsy to diagnose osteomyelitis. J Bone Joint Surg Br 1994; 76(02):311-314

16 Agarwal PK, Goel MM, Chandra T, Agarwal S. Predictive value of fine needle aspiration cytology of bone lesions. Acta Cytol 1997; 41(03):659-665

17 Barger AM. Cytology of bone. Vet Clin North Am Small Anim Pract 2017;47(01):71-84

18 Lew DP, Waldvogel FA. Osteomyelitis. N Engl J Med 1997;336(14): 999-1007

19 Siqueira EGM, Rahal SC, Ribeiro MG, Paes AC, Listoni FP, Vassalo FG. Exogenous bacterial osteomyelitis in 52 dogs: a retrospective study of etiology and in vitro antimicrobial susceptibility profile (2000-2013). Vet Q 2014;34(04):201-204

20 Griffiths GL, Bellenger CR. A retrospective study of osteomyelitis in dogs and cats. Aust Vet J 1979;55(12):587-591

21 Resnick D, Sweet DE, Madewell JE. Osteonecrosis: Pathogenesis, diagnostic techniques, specific situations, and complications. In: Resnick D, Kransdorf MJ, eds. Bone and Joint Imaging. 3rd ed. Philadelphia: Elsevier Saunders; 2004:1067-1069

22 Murphey MD, Foreman KL, Klassen-Fischer MK, Fox MG, Chung EM Kransdorf MJ. From the radiologic pathology archives imaging of osteonecrosis: radiologic-pathologic correlation. Radiographics 2014;34(04):1003-1028

23 Thrall DE, Robertson ID. The thoracic limb. In: Thrall DE, Robertson ID, eds. Atlas of Normal Radiographic Anatomy and Anatomic Variants in the Dog and Cat. St Louis: Elsevier Saunders; 2011:69-98

24 Schnötzinger D. Anatomische und röntgenologische untersuchungen zum ligamentum interosseum antebrachii des hundes [dissertation]. Vienna: University of Veterinary Medicine; 1995 